\title{
A Case of Fibrillary Glomerulonephritis Associated with Thrombotic Microangiopathy and Anti-Glomerular Basement Membrane Antibody
}

\author{
Akishi Momose $^{a} \quad$ Taku Nakajima $^{a}$ Shigetoshi Chiba ${ }^{a}$ \\ Kenjirou Kumakawa ${ }^{a}$ Yasuo Shiraiwa ${ }^{a}$ Nobuhiro Sasaki ${ }^{b}$ \\ Kazuo Watanabe ${ }^{c}$ Etsuko Kitano $^{d}$ Mitiyo Hatanaka $^{d}$ Hajime Kitamura ${ }^{d}$ \\ Departments of a Urology, ${ }^{b}$ Nephrology and ' Pathology, Jusendo General Hospital, \\ Koriyama, and d Department of Medical Technology, Kobe Tokiwa College, Kobe, Japan
}

\section{Key Words}

Fibrillary glomerulonephritis · Thrombotic microangiopathy · Anti-glomerular basement

membrane antibody $\cdot$ Reversible posterior leukoencephalopathy syndrome $\cdot$ Plasmapheresis

\begin{abstract}
We present the first report of a case of fibrillary glomerulonephritis (FGN) associated with thrombotic microangiopathy (TMA) and anti-glomerular basement membrane antibody (antiGBM antibody). A 54-year-old man was admitted to our hospital for high fever and anuria. On the first hospital day, we initiated hemodialysis for renal dysfunction. Laboratory data revealed normocytic-normochromic anemia with schistocytes in the peripheral smear, thrombocytopenia, increased serum lactate dehydrogenase, decreased serum haptoglobin, and negative results for both direct and indirect Coombs tests. Based on these results, we diagnosed TMA. Assays conducted several days later indicated a disintegrin-like and metalloprotease with a thrombospondin motif 13 (ADAMTS13) activity of 31.6\%, and ADAMTS13 inhibitors were negative. We started plasma exchange using fresh frozen plasma and steroid pulse therapy. AntiGBM antibody was found to be positive. Renal biopsy showed FGN. Blood pressure rose on the 46th hospital day, and mild convulsions developed. Based on magnetic resonance imaging of the head, the patient was diagnosed with reversible posterior leukoencephalopathy syndrome. Hypertension persisted despite administration of multiple antihypertensive agents, and the patient experienced a sudden generalized seizure. Computed tomography of the head showed multiple cerebral hemorrhages. However, his blood pressure subsequently decreased and the platelet count increased. TMA remitted following 36 plasma exchange sessions, but renal function was not restored, and maintenance hemodialysis was continued. The patient was discharged on the 119th day of hospitalization. In conclusion, it was shown that TMA, FGN and anti-GBM antibody were closely related.


Momose et al.: A Case of Fibrillary Glomerulonephritis Associated with Thrombotic

Microangiopathy and Anti-Glomerular Basement Membrane Antibody

\section{Background}

According to the World Health Organization's classification of glomerular diseases [1], both thrombotic microangiopathy (TMA) [2] and fibrillary glomerulonephritis (FGN) [3] consist of glomerular lesions, the former in the context of a vascular disease and the latter in the setting of a metabolic disease. Anti-glomerular basement membrane (GBM) antibody glomerulonephritis is considered to be a glomerular nephritis of a systemic disease [4]. Here, we report an anti-GBM antibody-positive patient with FGN associated with TMA.

\section{Case Report}

Our patient was a 54-year-old male who presented with anuria, fever, and lower-leg edema. On June 3,2012, the patient visited the urology department of a local community hospital with macroscopic hematuria and dysuria. He was diagnosed with acute prostatitis and began treatment with levofloxacin hydrate, loxoprofen sodium hydrate, and cernitin pollen extract. However, his condition did not improve, and he subsequently developed lower extremity numbness, bilateral flank pain, and diarrhea, followed by anuria. On June 11, he visited another hospital in his community and was eventually transferred to our hospital on June 13 for inpatient treatment of possible acute kidney injury.

Physical examination on admission revealed the following: height $175 \mathrm{~cm}$; body weight $80 \mathrm{~kg}$; systolic and diastolic blood pressure values 179 and $102 \mathrm{~mm} \mathrm{Hg}$, respectively; pulse 98 beats/min (regular), and body temperature $37.9^{\circ} \mathrm{C}$. The patient had a normal mental status despite persistent headache. His palpebral conjunctivae indicated signs of anemia. Radiographic images showed no abnormal findings. Both lower limbs were edematous. The results of clinical laboratory tests obtained on admission are summarized in table 1. Urinalysis showed high protein levels $(3 \mathrm{~g} / \mathrm{l})$, blood $(+++)$, pyuria, and a fractional sodium excretion rate of $20.3 \%$. Hematological analyses of peripheral blood yielded a white blood cell count of 9.61 $\times 10^{9}$ cells/l (neutrophils $85.0 \%$, lymphocytes $8.5 \%$ ), a red blood cell count of $3.44 \times 10^{12}$ cells $/ \mathrm{l}$, a hemoglobin level of $100 \mathrm{~g} / \mathrm{l}$, the presence of fragmented red blood cells, and a platelet count of $90 \times 10^{9}$ cells $/ \mathrm{l}$. C-reactive protein levels were as high as $160,900 \mu \mathrm{g} / \mathrm{l}$. The fibrinogen degradation product level was $1.168 \mathrm{~g} / \mathrm{l}$, the prothrombin time was $45.6 \mathrm{~s}$, and the fibrinogen level was $4.1 \mathrm{~g} / \mathrm{l}$. These measurements resulted in a disseminated intravascular coagulation score of 6 points. Serum biochemistry tests yielded a blood urea nitrogen level of $58.1 \mathrm{mmol} / \mathrm{l}$ and a creatinine level of 2,044 $\mu \mathrm{mol} / \mathrm{l}$, suggesting significant renal impairment. The lactate dehydrogenase level was $765 \mathrm{IU} / \mathrm{l}$, and the haptoglobin level was below $1.2 \mu \mathrm{mol} / \mathrm{l}$. The patient had hypoproteinemia, as evidenced by a total serum protein level of $58 \mathrm{~g} / \mathrm{l}$ and an albumin level of $24 \mathrm{~g} / \mathrm{l}$. Serum samples were negative for anti-nuclear antibodies as well as myeloperoxidase- and proteinase 3-specific antineutrophil cytoplasmic autoantibodies, although anti-GBM antibody levels were high at 230 ELISA units. Stool examination was negative for occult blood, intestinal pathogens, and verotoxins. Plain chest radiographs revealed no alveolar hemorrhage or other abnormal signs. On interview, the patient reported a history of cervical disc herniation diagnosed at the age of 52 years. He had no noteworthy family history and was not taking any medications on a regular basis at the time of admission to our hospital.

Changes in platelet counts, lactate dehydrogenase levels, and mean blood pressure over time during the 119-day hospital stay are schematically represented in figure 1, along with major therapeutic interventions. Computed tomography (CT) imaging conducted on admission indicated renal atrophy and ascites, but no collapse of the inferior vena cava. These findings negated the possibility of prerenal and postrenal failure and suggested an acute 
Table 1. Results of laboratory examinations on admission
Momose et al.: A Case of Fibrillary Glomerulonephritis Associated with Thrombotic Microangiopathy and Anti-Glomerular Basement Membrane Antibody

\begin{tabular}{|c|c|}
\hline Blood cell count & $\begin{array}{l}\text { WBC } 9.61 \times 10^{9} / 1 \\
\text { Neu } 79 \% \\
\text { Eos } 1.6 \% \\
\text { Bas } 0.2 \% \\
\text { Lymph } 11.9 \% \\
\text { Mono } 7.3 \% \\
\text { RBC } 3.44 \times 10^{12} / 1 \\
\text { Hb } 100 \mathrm{~g} / 1 \\
\text { Plt } 90 \times 10^{9} / 1\end{array}$ \\
\hline Blood chemistry & $\begin{array}{l}\text { Na } 128.3 \mathrm{mmol} / \mathrm{l} \\
\text { K } 6.84 \mathrm{mmol} / \mathrm{l} \\
\text { Cl } 95.9 \mathrm{mmol} / \mathrm{l} \\
\text { T.P } 58 \mathrm{~g} / \mathrm{l} \\
\text { Alb } 24 \mathrm{~g} / \mathrm{l} \\
\text { BUN } 59.1 \mathrm{mmol} / \mathrm{l} \\
\text { Cr } 2,044 \mu \mathrm{mol} / \mathrm{l} \\
\text { UA } 797 \mu \mathrm{mol} / \mathrm{l} \\
\text { CRP } 160,900 \mu \mathrm{g} / \mathrm{l} \\
\text { Ca } 1.8 \mathrm{mmol} / \mathrm{l} \\
\text { P } 1.4 \mathrm{mmol} / \mathrm{l} \\
\text { CPK } 308 \mathrm{IU} / \mathrm{l} \\
\text { T-Chol } 3.1 \mathrm{mmol} / \mathrm{l} \\
\text { Amylase } 296 \mathrm{IU} / \mathrm{l} \\
\text { LDH } 765 \mathrm{IU} / \mathrm{l} \\
\text { T.Bil } 25.0 \mu \mathrm{mol} / \mathrm{l} \\
\text { I.Bil } 17.1 \mu \mathrm{mol} / \mathrm{l} \\
\text { AST } 24 \mathrm{IU} / \mathrm{l} \\
\text { ALT } 23 \mathrm{IU} / \mathrm{l}\end{array}$ \\
\hline Serology & $\begin{array}{l}\text { IgG } 12.1 \mathrm{~g} / \mathrm{l} \\
\text { IgA } 2.5 \mathrm{~g} / \mathrm{l} \\
\text { IgM } 0.8 \mathrm{~g} / \mathrm{l} \\
\mathrm{C}_{3} 0.89 \mathrm{~g} / \mathrm{l} \\
\mathrm{C}_{4} 0.46 \mathrm{~g} / \mathrm{l} \\
\mathrm{CH}_{50} 46.2 \mathrm{U} / \mathrm{ml} \\
\mathrm{ANA}<40(-) \\
\mathrm{MPO}-\mathrm{ANCA}<10 \mathrm{U} / \mathrm{ml} \\
\text { PR3-ANCA }<10 \mathrm{U} / \mathrm{ml} \\
\text { Anti-GBM antibodies } 230 \mathrm{EU} \\
\text { Haptoglobin } \leq 1.2 \mu \mathrm{mol} / \mathrm{l}\end{array}$ \\
\hline Stool examination & $\begin{array}{l}\text { Occult blood (-) } \\
\text { Verocytotoxin }(-)\end{array}$ \\
\hline
\end{tabular}

aggravation of chronic kidney disease rather than a typical acute kidney injury. Since the patient had not undergone regular medical checkups, it was not clear whether proteinuria had existed in the past. During the early hospitalization period, we administered $\gamma$-globulin, diuretics, and hemodialysis, under the assumption that the patient had either sepsis- or druginduced acute kidney injury. Peripheral blood smears revealed the presence of fragmented red blood cells (hospital day 2), suggesting hemolytic uremic syndrome (HUS) resulting from food poisoning caused by Escherichia coli 0-157:H7 or other pathogenic strains. However, the patient had no diarrhea and was negative for intestinal pathogens and verotoxins. Epistaxis developed on hospital day 4, platelet counts and hemoglobin levels decreased to $29 \times 10^{9}$ cells/l and $55 \mathrm{~g} / \mathrm{l}$, respectively, and the lactate dehydrogenase level rose to 1,253 IU/l. The patient's anemia was unresponsive to erythropoiesis-stimulating therapy, and frequent 


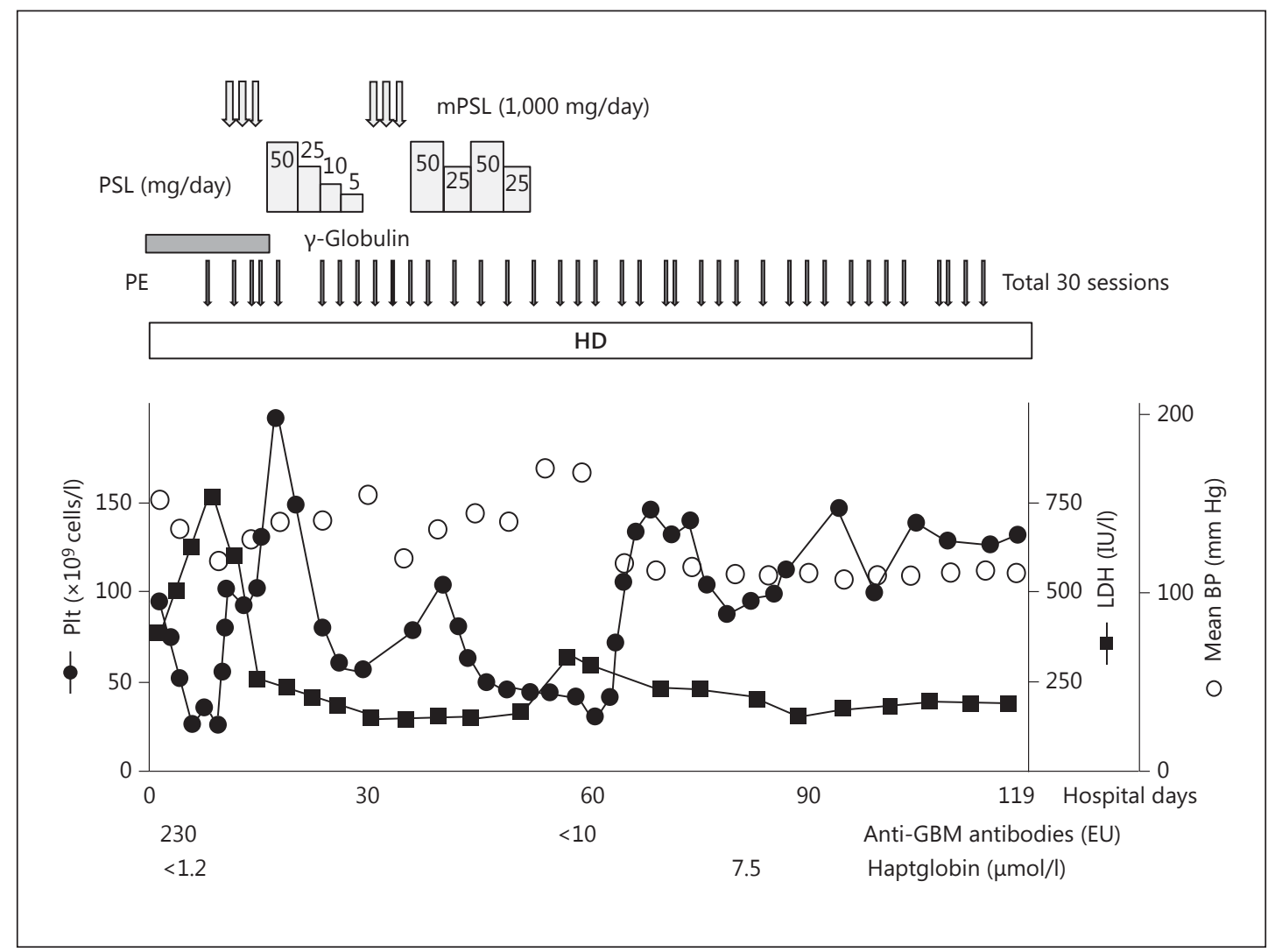

Fig. 1. Schematic presentation of therapies administered and changes in key clinical indices during the 119 days of hospitalization. $\mathrm{mPSL}=$ Methylprednisolone; $\mathrm{PSL}=$ prednisolone; $\mathrm{PE}=$ plasma exchange; $\mathrm{HD}=$ hemodialysis; Plt = platelets; $\mathrm{LDH}=$ lactate dehydrogenase; $\mathrm{BP}=$ blood pressure .

blood transfusions were required (i.e., a total of 16 units of irradiated red cell concentrates). His fever remained in the range of $38-39^{\circ} \mathrm{C}$ despite treatment with $\gamma$-globulin. The patient was diagnosed with TMA [thrombotic thrombocytopenic purpura (TTP) or HUS] based on the following findings: thrombocytopenia, hemolytic anemia evidenced by anemic signs and elevated lactate dehydrogenase levels, signs of renal impairment, fever, bleeding episodes (i.e., epistaxis), neuropsychiatric manifestations (i.e., headache), direct and indirect Coombs test results, and haptoglobin levels below $1.2 \mu \mathrm{mol} / \mathrm{l}$.

Assays conducted several days later indicated an ADAMTS13 activity of $31.6 \%$ (reference range 70-120\%), and ADAMTS13 inhibitors were negative. Serum factor $\mathrm{H}$ measured by the ELISA method was $0.473 \mathrm{~g} / \mathrm{l}$ (the standard value of factor $\mathrm{H}$ ranges from 0.3 to $0.6 \mathrm{~g} / \mathrm{l}$ ). These findings led us to administer plasma exchange therapy with a total of 30 units of fresh frozen plasma, starting on hospital day 8. On hospital day 15, steroid pulse therapy (3 methylprednisolone doses of $1,000 \mathrm{mg} /$ day) was initiated to lower the elevated anti-GBM antibody level. Following the completion of this 3-day course, the patient was given oral prednisolone at a starting dose of $50 \mathrm{mg} /$ day, which was gradually tapered and discontinued. After this, platelet counts rose to $197 \times 10^{9}$ cells/l, and kidney biopsy was conducted on hospital day 22 (fig. 2). Seventeen glomeruli examined by optic microscopy all showed destructive patterns with characteristic architecture ranging from atypical proliferative changes to global sclerosis. Renal arterioles, both afferent and efferent, showed extensive endothelial cell edema and swelling, indicative of glomeruloid changes. Renal tubules showed focal atrophy with massive 

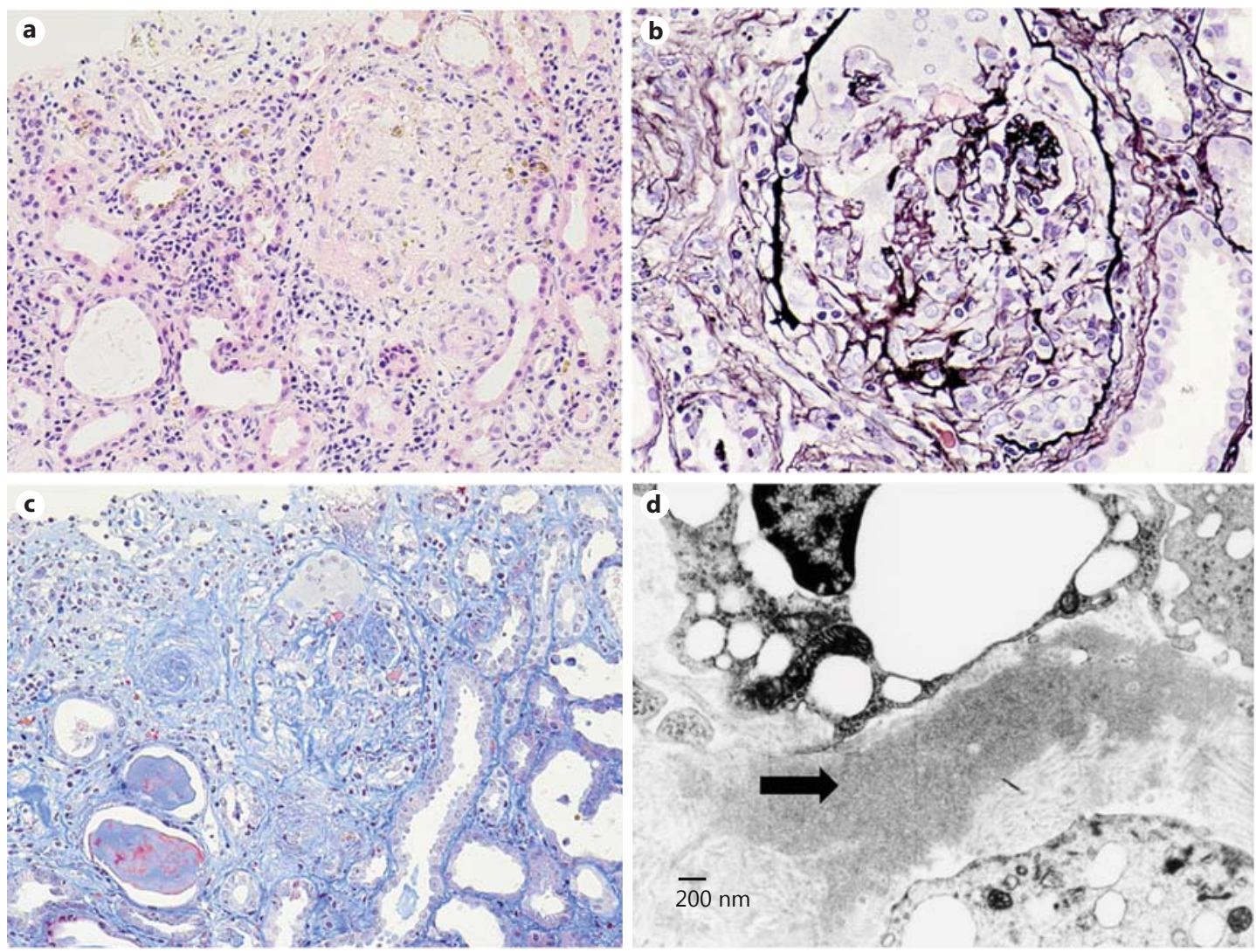

Fig. 2. Renal biopsy specimens examined on hospital day 22 using light microscopy. a Hematoxylin and eosin staining. Original magnification $\times 20$. b Periodic acid methenamine silver staining. Original magnification $\times 40$. c Metallothionein staining. Original magnification $\times 20$. d Electron microscopy. All 17 glomeruli examined under light microscopy show a loss of normal architecture. Renal arterioles, including afferent and efferent arterioles, show endothelial cell edema and proliferative changes, and atrophy of renal tubules. Electron microscopy demonstrates extensive, dense extracellular fibril deposits (arrow).

lymphocyte infiltration. Congo red staining of the biopsy specimen was negative. Electron microscopy showed that the loss of glomerular structure observed under optic microscopy was the result of an extensive and dense extracellular deposition of fibrillar components, which were larger in diameter than amyloid fibers and aggregated to form large bundles. Immunofluorescence analysis for immunoglobulin $\mathrm{G}$ and third component $\left(\mathrm{C}_{3}\right)$ deposits was not carried out because of glomerular collapse. Available findings supported the diagnosis of FGN involving severe glomerular destruction. On hospital day 29, a second course of steroid pulse therapy was started due to significantly decreased platelet counts.

On hospital day 46, the mean blood pressure values started to increase gradually. Although the target body weight was lowered and arotinolol hydrochloride and methyldopa hydrate were added to nifedipine and candesartan cilexetil, these measures did not achieve successful blood pressure control.

On hospital day 56 , the patient had a mild seizure. $\mathrm{T}_{2}$-weighted fluid-attenuated inversion recovery (FLAIR) magnetic resonance scans of the bilateral cerebellum, occipital lobe, and parietal lobe showed multiple high-intensity areas, suggesting a diagnosis of reversible posterior leukoencephalopathy syndrome (fig. 3a). Despite antihypertensive therapy with imidapril hydrochloride, carvedilol, nifedipine, and nitroglycerin infusion, hypertension 
Momose et al.: A Case of Fibrillary Glomerulonephritis Associated with Thrombotic Microangiopathy and Anti-Glomerular Basement Membrane Antibody
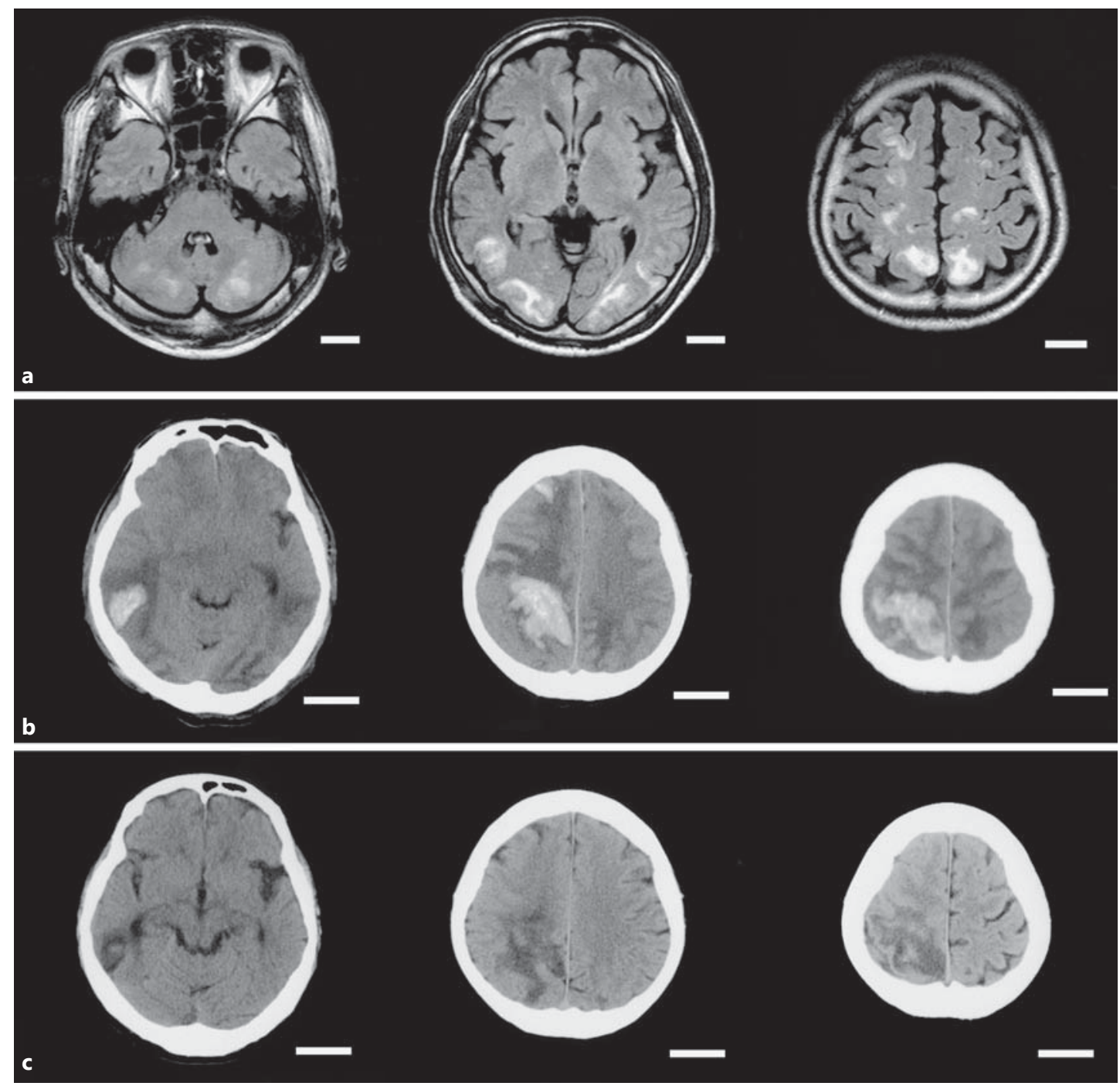

Fig. 3. Axial head magnetic resonance and computed tomography scans. a Fluid-attenuated inversion recovery scans taken on hospital day 48, when the patient complained of hand numbness. Magnetic resonance imaging shows multiple high-intensity areas in the cerebellum, occipital lobe, and parietal lobe. Magnetic resonance angiography reveals no significant stenosis of the major cerebral arteries. $\mathbf{b}$ Computed tomography scans taken after the onset of a generalized seizure on hospital day 61. Images demonstrate multiple hemorrhagic foci in the right hemisphere. Scans also depict an extension of the hemorrhage into the right lateral ventricle. c Computed tomography scans on hospital day 115 showing nearly complete absorption of the intracranial hematoma. Scale bars $=200 \mathrm{~mm}$.

persisted, and the patient's headache continued. On hospital day 61, generalized convulsions recurred, and head CT images showed multiple hemorrhagic lesions in the right temporal, right parietal, and right frontal lobes (fig. 3b). Doses of intravenous hemostatic medications were increased, while antihypertensive agents were administered nasogastrically. Blood pressure levels gradually decreased with the increase in platelet counts, starting around hospital day 65. On hospital day 72, the patient started rehabilitation exercises. The patient recovered enough to walk with a cane (fig. $3 \mathrm{c}$ ). He was transferred to a rehabilitation hospital on hospital day 119. 
Momose et al.: A Case of Fibrillary Glomerulonephritis Associated with Thrombotic Microangiopathy and Anti-Glomerular Basement Membrane Antibody

\section{Discussion}

FGN and immunotactoid glomerulonephritis are characterized by electron-dense deposits of fibrillary structures in the glomerular extracellular matrix. For both conditions, optical microscopy of renal biopsies reveals membranoproliferative glomerulonephritis-like lesions, which often require electron microscopy for differentiation. FGN is characterized by randomly oriented nonbranching fibrils with a mean diameter of $15-25 \mathrm{~nm}$, a size comparatively larger than that of amyloid fibrils $(8-10 \mathrm{~nm})[5,6]$. In our case, although immunofluorescence analysis of immunoglobulin $\mathrm{G}$ and $\mathrm{C}_{3}$ was not conducted because of glomerular collapse, the diagnosis of FGN was established based on the negative Congo red staining and electron microscopy findings.

The term TMA designates pathological conditions characterized by microangiopathic hemolytic anemia, thrombocytopenia resulting from peripheral destruction of platelets, and organ damage due to platelet thrombi (notably, renal impairment) [2]. The causes of TMA include drugs, malignant hypertension, and autoimmune diseases [2,7]. The differential diagnoses include disseminated intravascular coagulation syndrome, idiopathic thrombocytopenic purpura, heparin-induced thrombocytopenia, paroxysmal nocturnal hemoglobinuria, and systemic lupus erythematosus. Analyses of ADAMTS13 activity and ADAMTS13 inhibitors and further investigation suggested that idiopathic TTP or HUS, autoimmune diseases or malignant tumors were highly probable diagnoses [7]. Considering that ADAMTS13 activity and observed clinical signs did not meet the diagnostic criteria for atypical HUS [8], we judged that atypical TTP was more likely than atypical HUS.

A noteworthy finding was that our patient was positive for anti-GBM antibodies. These antibodies are autoantibodies specific for type IV collagen of the GBM. They are present at high levels in patients with anti-GBM nephritis and Goodpasture's syndrome, a condition often culminating in rapidly progressive glomerulonephritis [4]. The likelihood of Goodpasture's syndrome was rejected in our case because the patient did not present with any pulmonary disorders. While admitting the possibility that high levels of anti-GBM antibodies had a complicating impact on TMA or FGN, we considered it unlikely that anti-GBM nephritis was the primary cause given the renal biopsy findings. With all of the above taken into consideration, we concluded that the patient had FGN associated with TMA and high levels of anti-GBM antibodies.

Our literature search identified a patient with FGN who was diagnosed as having anti-GBM glomerulonephritis based on immunofluorescence microscopy findings [9]. This patient was found to be negative for circulating anti-GBM antibodies 1 week after the start of treatment. This example illustrates the possibility that the anti-GBM antibody status could shift from positive to negative over time in patients with anti-GBM nephritis, suggesting that anti-GBM antibodies may have aggravated the FGN in our patient.

A literature search conducted in 2011 identified 11 reported cases of TMA patients positive for anti-GBM antibodies [10]. The pathogenesis of TMA includes extensive endothelial injury from vasculitis [2]. Due to TMA-induced glomerular collapse, we did not attempt to diagnose anti-GBM nephritis via immunofluoroscopy examination of immunoglobulin G or $\mathrm{C}_{3}$ depositions. The fact that our patient was positive for anti-GBM antibodies may suggest that he had anti-GBM nephritis.

Factor $\mathrm{H}$ is a soluble complement regulator circulating in human plasma. Deficiency of factor $\mathrm{H}$ induces uncontrolled activation of the alternative pathway and $\mathrm{C}_{3}$ consumption, resulting in endothelial injury. Research suggests that factor $\mathrm{H}$ mutations confer a predisposition to the development of HUS [11], and factor $\mathrm{H}$ deficiency has been reported in anecdotal cases of FGN [12] and membranoproliferative glomerulonephritis type II [13]. These findings suggest that decreased levels of factor $\mathrm{H}$ contributed to the co-occurrence of FGN and TMA. 
Momose et al.: A Case of Fibrillary Glomerulonephritis Associated with Thrombotic

Microangiopathy and Anti-Glomerular Basement Membrane Antibody

However, the direct relation of FGN and TMA through factor $\mathrm{H}$ was not accepted because the factor $\mathrm{H}$ was within normal levels in our case. There may be a relationship between FGN and TMA through anti-GBM antibody in our case.

Reversible posterior leukoencephalopathy syndrome (RPLS) is characterized by neurologic conditions resulting from hypertensive cerebral edema [14]. To the best of our knowledge, 6 cases of antineutrophil cytoplasmic autoantibody-negative anti-GBM disease with central nervous system involvement have been reported in the literature [15]. It is likely that RPLS was caused by hypertension, which developed from the combination of anti-GBM antibodyand TMA-induced vasculitis, judging from uncontrolled rises in blood pressure and the onset of numbness and seizures. Our medical opinion was supported by a retrospective analysis of 46 acute TTP patients by Burrus et al. [16], which showed that renal function was the only variable highly associated with the occurrence of RPLS on brain magnetic resonance imaging in acute TTP patients.

Plasma exchange therapy can effectively improve atypical TTP through the following mechanisms: elimination of ultralarge von Willebrand factor multimers; supplementation of normal-size von Willebrand factor multimers, which play an important role in blood coagulation; removal of proinflammatory cytokines from the circulation; elimination of ADAMTS13 inhibitors, and supplementation with ADAMTS13. A previous study reported a case of FGN in which repeat plasma exchange therapy resulted in remission of refractory nephrotic syndrome [17]. These previous findings suggest that plasma exchange therapy contributed to improving both TMA and FGN in our case.

\section{Disclosure Statement}

No conflicts of interest are reported.

\section{References}

1 Churg J, Bernstein J, Glassock RJ: Renal Disease. Classification and Atlas of Glomerular Diseases, ed 2. Tokyo/ New York, Igakushoin, 1995.

-2 Moake JL: Thrombotic microangiopathies. N Engl J Med 2002;347:83-92.

3 Lui SL, Chan KW, Li FK, Chan TM, Cheng IK: Fibrillary glomerulonephritis: a case report. Hong Kong Med 1995 ; 1:69-71.

-4 Hudson BG, Tryggvason K, Sundaramoorthy M, Neilson EG: Alport's syndrome, Goodpasture's syndrome, and type IV collagen. New Engl J Med 2003;348:2543-2556.

5 Brady HR: Fibrillary glomerulopathy. Kidney Int 1998;53:1421-1429.

6 Ivanyi F, Degrell P: Fibrillary glomerulonephritis and immunotactoid glomerulopathy. Nephrol Dial Transplant 2004;19:2166-2170.

-7 Fujimura Y, Matsumoto M: Registry of 919 patients with thrombotic microangiopathies across Japan: database of Nara Medical University during 1998-2008. Inter Med 2010;49:7-15.

8 Nester CM, Thomas CP: Atypical hemolytic uremic syndrome: what is it, how is it diagnosed, and how is it treated? Hematology 2012;2012:617-625.

-9 Nilajgi S, Killen JP, Baer R, Renaut P, Mantha M: Fibrillary glomerulonephritis. NDT Plus 2011;4:413-415.

10 Torok N, Niazi M, Al Ahwel Y, Taleb M, Taji J, Assaly R: Thrombotic thrombocytopenic purpura associated with anti-glomerular basement membrane disease. Nephrol Dial Transplant 2010;25:3446-3449.

11 Meri S: Complement activation in diseases presenting with thrombotic microangiopathy. Eur J Intern Med 2013;24:496-502.

12 Bircan Z, Toprak D, Kilicaslan I, Solakoglu S, Uysal V, Ponard D, Turker G: Factor H deficiency and fibrillary glomerulopathy. Nephrol Dial Transplant 2004;19:727-730.

$\$ 13$ Pickering MC, Cook HT: Translational mini-review series on complement factor H: renal diseases associated with complement factor: novel insights from humans and animals. Clin Exp Immunol 2008;151:210-230. 
Momose et al.: A Case of Fibrillary Glomerulonephritis Associated with Thrombotic Microangiopathy and Anti-Glomerular Basement Membrane Antibody

$\$ 14$ Hinchey J, Chaves C, Appignani B, Breen J, Pao L, Wang A, Pessin MS, Lamy C, Mas JL, Caplan LR: A reversible posterior leukoencephalopathy syndrome. N Engl J Med 1996;334:494-500.

15 Gittins N, Basu A, Eyre J, Gholkar A, Moghal N: Cerebral vasculitis in a teenager with Goodpasture's syndrome. Nephrol Dial Transplant 2004;19:3168-3171.

16 Burrus TM, Mandrekar J, Wijdicks EM, Rabinstein AA: Renal failure and posterior reversible encephalopathy syndrome in patients with thrombotic thrombocytopenic purpura. Arch Neurol 2010;67:831-834.

17 Pliquett RU, Mohr P, El Din Mukhtar B, Girndt M, Markau S: Plasmapheresis leading to remission of refractory nephrotic syndrome due to fibrillary glomerulonephritis. J Med Case Rep 2012;6:116. 